\title{
Conspicuous Ethics: Existence of Price Dependent Ethical Utility Functions
}

\section{Pascal Stiefenhofer}

Newcastle University Business School, Newcastle upon Tyne, UK

Email: pascal.stiefenhofer@newcastle.ac.uk

How to cite this paper: Stiefenhofer, P. (2021) Conspicuous Ethics: Existence of Price Dependent Ethical Utility Functions. Applied Mathematics, 12, 252-261. https://doi.org/10.4236/am.2021.124016

Received: March 4, 2021

Accepted: April 5, 2021

Published: April 8, 2021

Copyright $\odot 2021$ by author(s) and Scientific Research Publishing Inc. This work is licensed under the Creative Commons Attribution International License (CC BY 4.0).

http://creativecommons.org/licenses/by/4.0/

\begin{abstract}
The purpose of this paper is to model ethical consumption behaviour based on price-dependent preferences. We motivate ethical consumption founded on the idea that ethical consumers compete for moral superiority relative to their peers by purchasing more expensive ethical conspicuous goods. An ethical consumer model in which consumers display moral consumption choices by purchasing ethical conspicuous goods is developed. This requires defining ethical conspicuous goods, social labels, and formalizing a set of assumptions on price-dependent ethical preferences. We show that an ethical utility function exists without relying on the usual transitivity assumption. We utilise Blashke's Rolling Theorem to show our main result.
\end{abstract}

\section{Keywords}

Existence, Balshke's Rolling Thoerem, Ethical Utility

\section{Introduction}

In "The Theory of the Leisure Class", Veblen ([1], chapter 4, p.35-36) recognizes that the consumption of "more excellent goods" is evidence of wealth and that failure to do so becomes a mark of inferiority and demerit. Veblen's theory of individual consumption is a theory based on individuals maximizing "esteem" by displaying wealth in order to distinguish themselves from other members of society. On a macroeconomic level, he describes the evolution of a society (social classes) based on the idea that individuals carve for esteem through the consumption of conspicuous goods. Veblen argues that a utility function, which, is independent of prices is not a suitable framework for the analysis of consumption behavior of conspicuous goods. Implicit in his formulation of the consumer, he believes that prices do not only signal the scarcity of goods in the coordina-

"More excellent goods" refers to product quality and luxury goods in this context. 
tion of consumption allocations, but they also affect the individual's preferences. Veblen's theory is one of the most influential critiques of the neoclassical economic model, partially criticizing it for its restriction of the utility function to the characteristics of goods. Veblen's critique inspires a new development of price dependent utility function within the neoclassical model. His views were first analytically introduced in Samuelson ([2], p.119-121), who formulates a model of conspicuous consumption with price dependent utility functions. In this model, Samuelson considers a price-parameterized utility function and a conspicuous good represented by gold. Samuelson's seminal work initiates two research directions of price dependent preferences. One research line continues to search for the motivation of price dependent preferences in the context of consumption of conspicuous goods, and the other research line focuses on the demand for money and other financial assets.

Here we pursue a new development of price dependent preferences in the context of ethically conspicuous goods. At variance to the existing literature on price dependent utility functions, we consider Veblen's original philosophy of esteem maximization, which yet lacks a rigorous formulation within the neoclassical model. The search for price dependent utility functions in the context of the neoclassical theory begins with Scitovsky [3] providing the rationale for taking prices as an index of product quality. He states that judging quality by its price is rational if consumers are informed about product qualities. However, Scitovsky does not establish the conditions of rational behavior, instead focusing on price effects, also known as Veblen effects, where prices and quantity demanded are positively correlated. Veblen effects are also analyzed in Leibstein [4] and Alcaly and Klevorick [5] with Kalman [6] and Pollak [7] being mathematically the most rigorous. This literature principally focuses on the motivation for price dependent utility functions and the derivation and implications of wealth effects by considering variations of the Slutsky equation. Price dependent utility functions have also been introduced into the "New Consumer Theory", also known as the Lancaster model [8], in the context of judging quality by price and snob appeal [5], where the price enters the utility as one of the characteristics of the goods. These papers establish analytically the conditions of positive correlation between prices and demand for goods.

In this paper, however, we are primarily interested in motivating ethical consumption by hypothesizing a society in which ethical consumers derive esteem from displaying their moral consumption choices. We show that a price dependent ethical utility function exists. In Section two we characterise ethical conspicuous goods and formalise the concept of a social label and the idea of conspicuous ethics. Section three introduces the axioms of rational ethical consumption behaviour and states the main results. Section four is a conclusion.

\section{The Ethical Consumption Model}

The theory on price dependent preferences is sparse. The various hypotheses 
motivating the study of price dependent preferences are: judging the quality of a good by its price [1], "Snob appeal" of a good [3], and treatment of money and other financial assets in temporal equilibrium models [2]. In this paper, we consider a new hypothesis for introducing price dependent preferences into consumer theory. In our model, prices perform the role of informing consumers about ethical production. We assume there exists a group of socially responsible consumers represented by an index $i=1, \cdots, m$ who do not only care about their own consumption of goods $l=1, \cdots, n$, with consumption bundle $x_{i} \in \mathbb{R}_{+}^{n}$, but they also care about certain ethical and social conditions $j=1, \cdots, k$ regarding production and distribution of these goods. Associated with a consumption bundle there is a price system $p \in \mathbb{R}_{+}^{n}$. Let the ethical consumer price $\mathfrak{p}$ be defined by the global market price $p_{M}$ plus a premium $p_{E}$ such that

$$
\mathfrak{p}=p_{M}+p_{E} \geq p_{\min },
$$

where $p_{\min }>0$ and $P_{E} \geq 0$ is a premium (R. Dragusanu and Nunn [9]), Figure 1 on p.219-220). It is assumed that the ethical price system $\mathfrak{p}=\left(\mathfrak{p}^{1}, \cdots, \mathfrak{p}^{n}\right) \in \mathbb{R}_{++}^{n}$ satisfies the normalization $\mathcal{S}:=\left\{\mathfrak{p} \in \mathbb{R}_{++}^{n}: \mathfrak{p}^{n}=1\right\}$ as the numeraire convention. This price normalization is useful in the calculation of derivatives. Moreover relative prices lead to demand functions which are homogeneous of degree zero. Such functions provide meaningful welfare analysis [7] [10]. In our model prices do not only signal the scarcity of resources in their efficient allocation process but also all these things our consumers care about regarding ethical production and distribution. Associated with an ethical price system $\mathfrak{p}$ there are $l=1, \cdots, n$ ethically produced and distributed consumption goods. We now characterize a consumption good $x_{i}^{l} \in \mathbb{R}_{+}$in terms of ethical values such as no child labor, environmental friendly production, minimum wages, safe work conditions, gender equality, human rights, etc. While some of these ethical values are quantifiable, others are clearly not. Hence, we introduce an information array $e^{l}$ collecting the words and symbols about production conditions associated with a good $x_{i}^{l}$. We assume that each good 1 is characterized by $j=1, \cdots, k$ ethical values $e^{l j}=\left(e^{l 1}, \cdots, e^{l k}\right)^{2}$. Each ethical value is indexed by $j \in 1, \cdots, k$ and is assumed to be known to an informed consumer. The collection of all ethical values associated with a consumption bundle $x_{i}$ is an information array given by

$$
e=\left(\begin{array}{ccc}
e^{11} & \cdots & e^{1 k} \\
\vdots & \ddots & \vdots \\
e^{n 1} & \cdots & e^{n k}
\end{array}\right) .
$$

It follows that a consumption good is characterized by a quantifiable physical property $x_{i}^{l}$ and an associated set of ethical values $e^{l}$, providing a description of production and distribution conditions regarding environment, health, gender, animal well-being, etc. An ethically produced good is hence denoted by ${ }^{2}$ Note that $e^{l}$ is not a quantity in $\mathbb{R}^{k}$ as in the Lancaster model, but a descriptive list of characteristics. Hence, it should not be confused with the mathematical definition of a vector, i.e., the inner product operation does not apply. In essence $e^{l}$ is a string of information. 
$\left.x_{i}^{l}\right|_{e^{l}} \in \mathbb{R}_{++}$. Note that $\left.x^{l}\right|_{e^{l}} \neq\left. x^{l}\right|_{e^{l^{\prime}}}$ for $e^{l} \neq e^{l^{\prime}}$. Our characterization of goods states that two physically identical goods produced under different ethical conditions are "nearly-identical" goods. The physically identical goods only differ in the way they are produced. Ethical consumers are informed consumers who can distinguish between such goods. A social label conveys this information to consumers. Our definition of a consumption good is a generalization of the characterization of goods by [11], which are defined by their physical, temporal, and spatial nature, to goods which also depend on a set of ethical values. We denote an ethical consumption bundle $\left.x_{i}\right|_{e}$ by

$$
\chi_{i} \in \mathcal{X}_{i}
$$

where $\mathcal{X}_{i} \subseteq \mathbb{R}_{+}^{n}$ is the consumption space characterized by ethical attributes $e$. We label goods $\chi_{i} \in \mathcal{X}_{i}$ satisfying such characteristics as ethical conspicuous goods. We formalize the idea of conspicuous goods in the subsequent three definitions.

Definition 1 (Ethical conspicuous goods). An ethical conspicuous consumption bundle $\chi_{i} \in \mathcal{X}_{i}$ is defined by a consumption bundle $x_{i} \in \mathbb{R}_{+}^{n}$ characterized by an array of descriptive ethical values $e$.

On the production side producers seek for social label certification in order to convey information about ethical production and distribution standards. Using (1) and (3) we introduce a definition of a voluntary social label that is based on a price signal $\mathfrak{p}\left(p_{M}, p_{E}\right)$ which informs consumers $i=1, \cdots, m$ about ethical production and distribution processes, and a class of ethically characterized commodities $\chi_{i} \in \mathcal{X}_{i}$, which further informs consumers descriptively about the way goods are produced.

Definition 2 (Voluntary social label). A voluntary social label $\mathfrak{S}$ is defined by a pair $\left(\mathfrak{p}, \chi_{i}\right)$ consisting of an ethical price system $\mathfrak{p} \in \mathcal{S}$ and commodity bundle $x_{i} \in \mathbb{R}^{n}$ characterized by a set of ethical standards e represented in words and symbols, which, is intended to inform consumers about ethical production and distribution standards. A consumer $i=1, \cdots, m$ who's consumption choices are informed by a social label $\mathfrak{S}$, i.e.,

$$
\mathfrak{S}_{i}:=\left(\mathfrak{p}, \chi_{i}\right) \in \mathcal{S} \times \mathcal{X}_{i}
$$

belongs to the class of ethical consumers.

A voluntary social label $\mathfrak{S}_{i}$ consists of an ethical price and ethical conspicuous commodity pair $\left(\mathfrak{p}, \chi_{i}\right)$ which belongs to the set $\mathcal{S} \times \mathcal{X}_{i}$. Definition 2 allows us to consider a group of informed consumers, where $\mathfrak{S}_{i}$ represents that $\mathfrak{S}$ is known to $i=1, \cdots, m$. These consumers have ethical preferences in the sense that they care about the way goods are produced and distributed. Consumers satisfying definition 2 are classified as ethical consumers. It remains to characterize their consumption behavior. We begin by motivating ethical consumption behavior.

Definition 3 (Conspicuous ethics). Conspicuous ethics refers to an ethical consumption behavior where an informed consumer $\mathfrak{S}_{i}$, for $i=1, \cdots, n$, ex- 
presses superior ethical responsibility towards society relative to other members of society by purchasing ethically produced and distributed goods $\chi_{i}$ at ethical prices $\mathfrak{p}$. It is assumed that ethical consumers derive esteem from displaying their social responsibility through the consumption of ethically conspicuous goods. Positive ethical consumption is satisfied for all pairs

$$
\left(\mathfrak{p}, \chi_{i}\right) \in \mathfrak{S}_{i} \text {. }
$$

An ethical consumer also expresses superior ethical responsibility towards society by boycotting the consumption of unethically produced and distributed goods. Negative ethical consumption is satisfied if

$$
\text { either } p \neq \mathfrak{p} \text { or } x_{i} \neq \chi_{i} \text {. }
$$

Ethical consumers base their consumption decisions on the basis of ethical values, such as human rights, environmentally friendly and sustainable production, and animal well-being. We hypothesize that they express moral superiority towards society via positive and negative consumption of ethical conspicuous goods. We consider ethical consumers who derive esteem from the consumption of "more expensive" ethical conspicuous goods. This relates our model to Veblen [1] who discusses the evolution of society based on the competition of esteem. Veblen also introduces the idea of price dependent preferences. This is similar to our model, the motivation for priced dependent preferences, however, is different and an existence theorem is absent.

We characterize the consumer's preference ordering $\succeq_{q}$ by an ethical price index $q$ associated with an ethical market price price $\mathfrak{p}$. It is assumed that the price depending preference ordering $\succeq_{q}$ is unaffected by a proportional change in all prices $\mathfrak{p}$ [7]. Moreover, we consider normalized prices $q$, hence, our characterization of the consumer preference is at variance to Kalman, who assumes a preference ordering of the form $\succeq_{p}$ over a consumption-market price space $(x, p) \in \mathbb{R}_{+}^{n} \times \mathbb{R}_{++}^{n}$, where $x$ is a commodity bundle ([6]: A.1, p.498). Stiefenhofer and Zhang [12] consider a class of price dependent ethical utility functions.

We want to represent preferences, which do not only depend on ethical consumption bundles but also on relative prices, which inform consumers about the way goods are produced. We are going to consider utility functions of the form $u_{i}\left(\chi_{i}, q\right)$. Let the open normalized price simplex be denoted by

$$
\mathbb{S}:=\left\{\mathfrak{p} \in \mathbb{R}_{++}^{n}: \sum_{k=1}^{n} \mathfrak{p}^{k}=1\right\},
$$

with its closure simplex defined by

$$
\overline{\mathbb{S}}:=\left\{\mathfrak{p} \in \mathbb{R}_{+}^{n}: \sum_{k=1}^{n} \mathfrak{p}^{k}=1\right\} .
$$

We now formalize the hypothesis that consumers do not only have preferences for the physical properties of goods, but they also care about the way goods are produced. Hence by (3) and (8) we defined a preference relation of the form $\succeq_{(q, \chi)}$ over the preference ordering space $\left(q, \chi_{i}\right) \in \overline{\mathbb{S}} \times \mathcal{X}_{i}$, where 


$$
u_{i}: \overline{\mathbb{S}} \times \mathcal{X}_{i} \rightarrow \mathbb{R}
$$

is the price-dependent ethical utility function. Stiefenhofer [13] establishes the geometric conditions of smooth ethical utility functions. Stiefenhofer and Zhang [12] provide an example of a price dependent ethical utility function. We can now formulate an ethical consumption model, where production is implicitly formulated through a reallocation of initial endowments. Let an ethical consumer $i=1, \cdots, m$ be endowed with a vector of initial endowments

$\left.\omega_{i}\right|_{e}=\left.\left(\left.\omega_{i}^{1}\right|_{e^{1}}, \cdots,\left.\omega_{i}^{n}\right|_{e^{n}}\right) \in \Omega_{i}\right|_{e} \subseteq \mathbb{R}_{++}^{n}$. We denote $\left.\omega_{i}\right|_{e}$ by $\omega_{i}$ and $\left.\Omega_{i}\right|_{e}$ by $\Omega_{i}{ }^{3}$.

Definition 4. The disentanglement of the ethical commodity market price $\mathfrak{p}$ into a price index $q$ is given by a map

$$
s: \mathcal{S} \rightarrow \mathbb{S}
$$

defined by $s(\mathfrak{p}):=\frac{\mathfrak{p}^{k}}{\sum_{k=1}^{n} \mathfrak{p}^{k}}$ for $k=1, \cdots, n$.

\section{Existence of an Ethical Utility Function}

Assumption 1 (Ethical preferences).

a) (i) For all $\left.x\right|_{e, \mathfrak{p}},\left.y\right|_{e, \mathfrak{p}}$, either $\left.\left.x\right|_{e} \succeq_{\mathfrak{p}} y\right|_{e}$ or $\left.\left.y\right|_{e} \succeq_{\mathfrak{p}} x\right|_{e}$. (ii) $\left.x\right|_{e, \mathfrak{p}},\left.y\right|_{e, \mathfrak{p}},\left.z\right|_{e, \mathfrak{p}}$ with $\left.x\right|_{e, \mathfrak{p}} \neq\left. z\right|_{e, \mathfrak{p}}$ implies $\left.\alpha x\right|_{e}+\left.\left.(1-\alpha) y\right|_{e} \succ_{p} y\right|_{e}$ for $0<\alpha<1$. (iii) The preference relation $\succeq_{\mathfrak{p}}$ is continuous in $\left.x\right|_{e}$ and $\mathfrak{p} \in \mathcal{S}$. (non-transitivity, Sonnenschein (1971).

b) If $\left.\left.x\right|_{e} \succeq_{\mathfrak{p}} y\right|_{e}$ then $\left.\left.x\right|_{e} \succeq_{\mathfrak{p}, \lambda} y\right|_{e}$ for all $\lambda>0$, (preference orderings depend on relative prices).

c) (i) $\left.\left.\left.\left.x\right|_{e} \succ_{\mathfrak{p}} y\right|_{e} \Rightarrow x\right|_{e} \succ_{p_{F T}^{*}} y\right|_{e}$, and (ii) $\left.\left.\left.\left.x\right|_{e} \sim_{\mathfrak{p}} y\right|_{e} \Rightarrow x\right|_{e} \succeq_{p_{F T}^{*}} y\right|_{e} \quad$ (nonnegative price effect on preferences).

(a) (iii) Similarly to Sonnenschein [14] we introduce the concept of price-continuous preferences. We follow Sonnenschein's approach, which, together with a strict convexity assumption (ii) and a completeness assumption (i) on the price dependent preference relation is sufficient to establish rational behavior in the consumption space $\mathcal{X}_{i}$. Hence, in our model of ethical consumption, we are able to dispense of the usual transitivity condition. (b) states that preferences depend on a relative price system. This is at variance to Kalman [6]. However, a similar condition with its welfare implications is also discussed in Pollak [7]. (c) is a very specific assumption which allows modeling of conspicuous consumption of ethically produced goods. It allows for a positive correlation between prices and demand for ethical consumption goods and the boycott of unethically produced goods.

The assumptions 1 allow us to model a social label $S_{i}$ as introduced in definition 2 in the utility of the ethical consumer. First, we observe that by the characterization of consumption goods through the characterization of $\mathcal{X}_{i}$ ethical values enter the utility of the consumer through a characterization of ethical preferences distributed over an ethical consumption space. Second, the price ${ }^{3} \mathrm{We}$ do not intend to explicitly model ethical production in this paper. We are, however, aware that this is another interesting problem. 
signal provided by the ethical market price $\mathfrak{p}$ enters the utility of the consumer through the assumption on the preference relation allowing for the positive correlation between the ethical price signal and demand for ethical consumption goods. We now introduce our model.

Definition 5 (The ethical consumer model). Consider a group of informed consumers $\mathfrak{S}_{i}$, for $i=1, \cdots, m$ satisfying definition 2 . Let an ethical consumer $i=1, \cdots, m$ be endowed with a vector of initial endowments $\omega_{i} \in \Omega_{i}$, and let his set of feasible consumption allocations be defined by

$\mathcal{B}_{i}\left(\mathfrak{p}, \omega_{i}\right):=\left\{\left(\mathfrak{p}, \chi_{i}\right) \in \mathcal{S} \times \mathbb{R}_{+}^{n}: \mathfrak{p} \cdot \chi_{i} \leq \mathfrak{p} \cdot \omega_{i}\right\}$. Then for any ethical market price system $\mathfrak{p} \in \mathcal{S}$ and price disentanglement $s(\mathfrak{p})$ into an ethical price index $\mathrm{q}$ given in definition 4 , the objective of the consumer is formalized as

$$
\underset{\chi_{i} \in \mathcal{B}_{i}\left(\mathfrak{p}, \omega_{i}\right)}{\operatorname{argmax}} u_{i}\left(\chi_{i} ; s(\mathfrak{p}), \mathfrak{p}\right) .
$$

Lemma 2. The indifference set $\mathcal{I}\left(\sim_{\mathfrak{p}}\right):=\left\{\left(\left.x\right|_{\mathfrak{p}},\left.y\right|_{\mathfrak{p}}\right) \in \mathbb{X} \times \mathbb{S}: x \succeq_{\mathfrak{p}} y \cap y \succeq_{\mathfrak{p}} x\right\}$, where $\mathbb{X} \subseteq \mathbb{R}_{+}^{n} \times \mathbb{R}_{+}^{n}$ is closed.

From now, we simplify notation where not confusion arises. We assume that prices and ethically characterized goods are understood from the context.

Proof. We decompose the indifference set $\mathcal{I}$ into the two sets $A\left(\succeq_{\mathfrak{p}}\right)$ and $A\left(\preceq_{p}\right)$ where

$$
A\left(\succeq_{\mathfrak{p}}\right):=\left\{\left(\left.x\right|_{\mathfrak{p}},\left.y\right|_{\mathfrak{p}}\right) \in \mathbb{X} \times \mathbb{S}: x \succeq_{\mathfrak{p}} y\right\}
$$

is a consumption bundle pair allocation set associated with the price dependent preference relation $\succeq_{p}$ and similarly

$$
A\left(\preceq_{\mathfrak{p}}\right):=\left\{\left(\left.x\right|_{\mathfrak{p}},\left.y\right|_{\mathfrak{p}}\right) \in \mathbb{X} \times \mathbb{S}: x \preceq_{\mathfrak{p}} y\right\}
$$

is associated with the price dependent preference $\preceq_{\mathfrak{p}}$.

Step 1: Pick any point $\left(\left.\hat{x}\right|_{\mathfrak{p}},\left.\hat{y}\right|_{\mathfrak{p}}\right)$ not in $A\left(\succeq_{\mathfrak{p}}\right)$. Then there is a set

$$
B\left(\succ_{\mathfrak{p}}\right):=\left\{\left(\left.x\right|_{\mathfrak{p}},\left.y\right|_{\mathfrak{p}}\right) \in \mathbb{X} \times \mathbb{S}: y \succeq_{\mathfrak{p}} x \cap x \nsucceq_{\mathfrak{p}} y\right\}
$$

and a set

$$
\hat{B}\left(\succ_{\mathfrak{p}}\right):=\left\{\left.y\right|_{\mathfrak{p}} \in \mathbb{R}_{+}^{n} \times \mathbb{S}: y \succeq_{p} x \bigcap x \nsucceq_{\mathfrak{p}} y\right\}
$$

associated with $\succeq_{\mathfrak{p}}$ such that $\left.\hat{y}\right|_{\mathfrak{p}} \in \hat{B}\left(\succ_{\mathfrak{p}}\right)$. By openness of the set $\hat{B}\left(\succ_{\mathfrak{p}}\right)$ we can construct a neighborhood $\mathcal{B}_{\left(\left.\hat{y}\right|_{\mathfrak{p}}, \varepsilon\right)}=\left\|\left.y\right|_{\mathfrak{p}}-\left.\hat{y}\right|_{\mathfrak{p}}\right\| \leq \varepsilon$ for small $\varepsilon \in \mathbb{R}_{++}$. By Blaschke's theorems ([15], p.113-117), we now show that $\mathcal{B}_{\left(\left.\hat{y}\right|_{p}, \varepsilon\right)}$ is the largest set contained in some finite convex set in $\hat{B}\left(\succ_{\mathfrak{p}}\right)$. The theorems imply a construction of a regularized convex set in $\hat{B}\left(\succ_{\mathfrak{p}}\right)$ by

$$
\operatorname{Conv}\left(f_{1}\left(\left.y\right|_{\mathfrak{p}} ^{1}\right), \cdots, f_{m}\left(\left.y\right|_{\mathfrak{p}} ^{m}\right)\right)=\left\{\sum_{i=1}^{m} \lambda^{i} f_{i}\left(\left.y\right|_{\mathfrak{p}} ^{i}\right): \lambda^{i} \geq 0, \sum_{i}^{m} \lambda_{i}=1\right\}
$$

where $f$ is smooth and convex function with the image of $\sum_{i}^{m} f_{i}$ complete. Blaschke's rolling theorems then imply there exists a largest $S \subset \operatorname{Conv}\left(f_{1}\left(\left.y\right|_{\mathfrak{p}} ^{1}\right), \cdots, f_{m}\left(\left.y\right|_{\mathfrak{p}} ^{m}\right)\right)$ with radius $r=(\sup k)^{-1}$ where $k$ is the curvature such that $k(S)<k\left(\operatorname{Conv}\left(f_{1}\left(\left.y\right|_{\mathfrak{p}} ^{1}\right), \cdots, f_{m}\left(\left.y\right|_{\mathfrak{p}} ^{m}\right)\right)\right)$ at some point $P$. Hence 
we choose $\varepsilon \leq r$ and obtain

$$
\mathcal{B}_{\left(\left.\hat{y}\right|_{\mathfrak{p}}, \varepsilon\right)} \subset S_{y} \subset \hat{B}\left(\succ_{\mathfrak{p}}\right)
$$

For each $i$ we have that $f_{i}\left(\left.y\right|_{\mathfrak{p}} ^{i}\right) \in\left\{\left.y\right|_{\mathfrak{p}} \in \mathbb{R}_{+}^{n} \times \mathbb{S}: y \succeq_{\mathfrak{p}} \hat{x} \cap \hat{x} \Varangle_{\mathfrak{p}} y\right\}$. Alternatively we have that $\left.\hat{x}\right|_{\mathfrak{p}}$ belongs to an open set $C\left(\prec_{\mathfrak{p}}\right)=\left\{\left.x\right|_{\mathfrak{p}} \in \mathbb{R}_{+}^{n} \times \mathbb{S}: f_{i}\left(\left.y\right|_{\mathfrak{p}} ^{i}\right) \succ_{\mathfrak{p}} \hat{x}\right\}$. Then for each $i$ we construct a neighborhood $\mathcal{B}_{\left(\left.\hat{x}\right|_{\mathfrak{p}}, \varepsilon\right)}^{i}=\left\|\left.x\right|_{\mathfrak{p}}-\left.\hat{x}\right|_{\mathfrak{p}}\right\| \leq \varepsilon$ with center $\left.\hat{x}\right|_{\mathfrak{p}}$ and radius $\varepsilon \in \mathbb{R}_{++}$. By a similar construction as above using Blaschke's rolling theorems and $\bigcap_{i=1}^{m} \mathcal{B}_{\left(\hat{x}_{\mid}, \varepsilon\right)}^{i}=\mathcal{B}_{\left(\left.\hat{x}\right|_{p}, \varepsilon\right)}$ we obtain for all $i$

$$
\left.\hat{x}\right|_{\mathfrak{p}} \in \mathcal{B}_{\left(\hat{x}_{\mid}, \varepsilon\right)} \subset \mathcal{B}_{\left(\left.\hat{x}\right|_{\mathfrak{p}}, \varepsilon\right)}^{i} \subset S_{x} \subset C\left(\prec_{\mathfrak{p}}\right),
$$

which shows that for each $i$ we have that $f_{i}\left(\left.y\right|_{\mathfrak{p}} ^{i}\right) \in \hat{B}\left(\succ_{\mathfrak{p}}\right)$ by the strict convexity assumption. We construct

$$
\mathcal{B}\left(\left.\hat{x}\right|_{\mathfrak{p}},\left.\hat{y}\right|_{\mathfrak{p}}\right)=\mathcal{B}_{\left(\left.\hat{x}\right|_{\mathfrak{p}}, \varepsilon\right)} \times \mathcal{B}_{\left(\left.\hat{y}\right|_{\mathfrak{p}}, \varepsilon\right)} .
$$

Consider any pair $\left(\left.x\right|_{\mathfrak{p}},\left.y\right|_{\mathfrak{p}}\right) \in \mathcal{B}\left(\left.\hat{x}\right|_{\mathfrak{p}},\left.\hat{y}\right|_{\mathfrak{p}}\right)$ the from above, we have that $\left.\hat{y}\right|_{\mathfrak{p}} \in \mathcal{B}_{\left(\hat{y}_{\mathfrak{p}}, \varepsilon\right)} \subset \hat{B}\left(\succ_{\mathfrak{p}}\right)$ and thus $(x, y)$ is not in $A\left(\succeq_{\mathfrak{p}}\right)$. It follows that $\mathcal{B}\left(\left.\hat{x}\right|_{\mathfrak{p}},\left.\hat{y}\right|_{\mathfrak{p}}\right)$ doe not satisfy $\succeq_{\mathfrak{p}}$. Need to show the opposite relation. This follows from a similar analysis as above. Since A and B are closed sets it follows that its intersection $\mathcal{I}$ is also closed. This completes the proof.

Theorem 3 (Existence of an esteem function). Let assumptions 1 hold. There exists a continuous price dependent ethical utility function $u_{i}: \mathcal{S} \times \mathbb{R}_{++}^{n} \rightarrow \mathbb{R}$, for all $\left(\mathfrak{p}, \chi_{i}\right) \in \mathfrak{S}_{i}$ and $i=1, \cdots, m$, such that if $\chi_{i}^{*} \in \mathcal{B}_{i}\left(\mathfrak{p}, w_{i}\right)$, then $u_{i}\left(\mathfrak{p}, \chi_{i}^{*}\right)=\max u_{i}\left(\mathfrak{p}, \chi_{i}\right)$ if and only if $\chi_{i}^{*}=f\left(\mathfrak{p}, s(\mathfrak{p}), w_{i}\right)$, where $\mathrm{f}$ is derived from $R(\mathfrak{p})$.

Proof. Step 1. We show that there exists a continuous mapping $k: \mathbb{X} \rightarrow \mathbb{R}$ derived from the preference relation $\succeq_{\mathfrak{p}}$.

Let $\mathbb{X}:=\mathbb{R}_{+}^{n} \times \mathbb{R}_{+}^{n} \times \mathbb{S}$ be a Banach space equipped with a quasi metric $d\left(\left.x\right|_{\mathfrak{p}},\left.y\right|_{\mathfrak{p}}\right):=\frac{\left.y\right|_{\mathfrak{p}}}{\left\|\left.y\right|_{\mathfrak{p}}\right\|}\left\|\left.x\right|_{\mathfrak{p}},\left.y\right|_{\mathfrak{p}}\right\|$. A consumer is indifferent for all points $\mathcal{I}:=R(\mathfrak{p}) \cap R^{-1}(\mathfrak{p})$. We now define

$$
m\left(\left.x\right|_{\mathfrak{p}},\left.y\right|_{\mathfrak{p}}\right):=\left(\left.x\right|_{\mathfrak{p}},\left.y\right|_{\mathfrak{p}}\right) \in \arg \min \frac{\left.y\right|_{\mathfrak{p}}}{\left\|\left.y\right|_{\mathfrak{p}}\right\|}\left\|\left(\left.x\right|_{\mathfrak{p}},\left.y\right|_{\mathfrak{p}}\right),\left(\left.z\right|_{\mathfrak{p}},\left.w\right|_{\mathfrak{p}}\right)\right\|
$$

for all $\left(\left.z\right|_{\mathfrak{p}},\left.w\right|_{\mathfrak{p}}\right) \in \mathcal{I} . \quad m\left(\left.x\right|_{\mathfrak{p}},\left.y\right|_{\mathfrak{p}}\right)$ is continues since $\mathcal{I}$ is a closed set. Closedness of $\mathcal{I}$ follows immediately from the continuity and closedness of $R(\mathfrak{p})$ and $R^{-1}(\mathfrak{p})$. Hence defining

$$
k\left(\left.x\right|_{\mathfrak{p}},\left.y\right|_{\mathfrak{p}}\right)=m\left(\left.x\right|_{\mathfrak{p}},\left.y\right|_{\mathfrak{p}}\right)
$$

it follows that the mapping $k$ is continuous. Moreover, it holds that for all $\left(\left.x\right|_{\mathfrak{p}},\left.y\right|_{\mathfrak{p}}\right) \in \mathbb{X} \backslash \mathcal{I}$ 


$$
k\left(\left.x\right|_{\mathfrak{p}},\left.y\right|_{\mathfrak{p}}\right) \neq 0 .
$$

(i) To show that $k\left(\left.x\right|_{\mathfrak{p}},\left.y\right|_{\mathfrak{p}}\right)>0$ we only need to restrict Equation (20) to point $\left.x\right|_{\mathfrak{p}} \in P(y)$. (ii) To show that $k\left(\left.x\right|_{\mathfrak{p}},\left.y\right|_{\mathfrak{p}}\right)<0$ we only need to restrict Equation (20) to point $\left.y\right|_{\mathfrak{p}} \in P(x)$. (iii) It follows that the restriction of Equation (20) for all points $\left(\left.x\right|_{\mathfrak{p}},\left.y\right|_{\mathfrak{p}}\right)$ not in $P(y) \times P(x)$ implies that

$$
k\left(\left.x\right|_{\mathfrak{p}},\left.y\right|_{\mathfrak{p}}\right)=0 \text {. }
$$

Hence this holds for all $\left(\left.x\right|_{\mathfrak{p}},\left.y\right|_{\mathfrak{p}}\right) \in \mathcal{I}$. (iv) We need to show that Equation (20) is a quasi metric. Hence, we translate the original coordinate system through the point $\left.x\right|_{\mathfrak{p}}$ such that under the translation $\left.x\right|_{\mathfrak{p}}$ is a null vector. Then it follows that Equation (20) generates negative values since $\frac{\left.y\right|_{\mathfrak{p}}}{\left\|\left.y\right|_{\mathfrak{p}}\right\|}=-1$.

Step 2. We show that a continuous parameterized utility function exists and that the model of the consumer is well defined. To show this let's denote by $B_{i}=\left\{\left.x\right|_{\mathfrak{p}} \in \mathbb{R}_{+}^{n} \times \mathbb{S}:\left.\mathfrak{p} \cdot x\right|_{\mathfrak{p}} \leq w_{i}\right\}$ the consumer's set of feasible consumption allocations and define

$$
u\left(\left.x\right|_{\mathfrak{p}} ; s(p)\right):=\min _{\left.y\right|_{\mathfrak{p}} \in B} k\left(\left.x\right|_{\mathfrak{p}},\left.y\right|_{\mathfrak{p}}\right)
$$

By continuity of $k$ and the construction of $B_{i}$ it follows that $u$ is continues. We can now formulate the model of the consumer.

We need to show that if $\left.x\right|_{\mathfrak{p}} ^{*} \in B_{i}(\mathfrak{p})$, then $u_{i}\left(\left.x\right|_{\mathfrak{p}} ^{*} ; s(\mathfrak{p})\right)=\max u_{i}\left(x,\left.x\right|_{\mathfrak{p}}\right)$ if and only if $\left.x\right|_{\mathfrak{p}} ^{*}=f\left(\mathfrak{p} ; s(\mathfrak{p}), w_{i}\right)$. Suppose that $\left.x\right|_{\mathfrak{p}} ^{*}=f\left(\mathfrak{p} ; s(\mathfrak{p}), w_{i}\right)$. Then it holds that $k\left(\left.x\right|_{\mathfrak{p}} ^{*},\left.y\right|_{\mathfrak{p}}\right) \geq 0$ for all $\left.y\right|_{\mathfrak{p}} \in B_{i}(\mathfrak{p})$ by step 1 . By definition 24 we obtain

$$
u\left(\left.x\right|_{\mathfrak{p}} ^{*} ; s(\mathfrak{p})\right):=\min _{\left.y\right|_{\mathfrak{p}} \in B} k\left(\left.x\right|_{\mathfrak{p}} ^{*},\left.y\right|_{\mathfrak{p}}\right)=0
$$

for all $\left.y\right|_{\mathfrak{p}}=\left.x\right|_{\mathfrak{p}} ^{*}$ since $k\left(\left.x\right|_{\mathfrak{p}} ^{*},\left.x\right|_{\mathfrak{p}} ^{*}\right)=0$ by step 1 . On contrary $\left.x\right|_{\mathfrak{p}} ^{*}$ maximizes $u_{i}\left(\left.x\right|_{\mathfrak{p}} ; s(\mathfrak{p})\right)$ for all $\left.x\right|_{\mathfrak{p}} \in B(\mathfrak{p})$ since

$$
u\left(\left.x\right|_{\mathfrak{p}} ; s(\mathfrak{p})\right):=\min _{\left.y\right|_{\mathfrak{p}} \in B} k\left(\left.x\right|_{\mathfrak{p}},\left.y\right|_{\mathfrak{p}}\right)=k\left(\left.x\right|_{\mathfrak{p}},\left.x\right|_{\mathfrak{p}}\right)=0 .
$$

Suppose $u\left(\left.x\right|_{\mathfrak{p}} ^{*} ; s(\mathfrak{p})\right)=\max _{x \in B(\mathfrak{p})} u\left(\left.x\right|_{\mathfrak{p}} ; s(\mathfrak{p})\right)$ and that $\left.x\right|_{\mathfrak{p}} ^{*} \in B(\mathfrak{p})$. We will show that $\left.x\right|_{\mathfrak{p}} ^{*}=\left.x\right|_{\mathfrak{p}} ^{* *}$, assuming that $\left.x\right|_{\mathfrak{p}} ^{* *}=f\left(\mathfrak{p} ; s(\mathfrak{p}), w_{i}\right)$. From above, since $\left.x\right|_{p} ^{* * *}=f(p ; s(p), m)$, we have that

$$
u\left(\left.x\right|_{\mathfrak{p}} ^{* *} ; s(\mathfrak{p})\right)=\max _{x \in B(\mathfrak{p})} u\left(\left.x\right|_{\mathfrak{p}} ^{*} ; s(\mathfrak{p})\right)=u\left(\left.x\right|_{\mathfrak{p}} ; s(\mathfrak{p})\right)=0 .
$$

It then follows that $k\left(\left.x\right|_{\mathfrak{p}} ^{*},\left.y\right|_{\mathfrak{p}}\right) \geq 0$ for each $\left.y\right|_{\mathfrak{p}} \in B(\mathfrak{p})$ and $k\left(\left.x\right|_{\mathfrak{p}} ^{* *},\left.y\right|_{\mathfrak{p}}\right) \geq 0$ for each $\left.y\right|_{\mathfrak{p}} \in B(\mathfrak{p})$. This can be reformulated as $\left.x\right|_{\mathfrak{p}} ^{*} \in R(y)$ and $\left.x\right|_{\mathfrak{p}} ^{* *} \in R(y)$ for each $\left.y\right|_{\mathfrak{p}} \in B(\mathfrak{p})$ so that both $\left.x\right|_{\mathfrak{p}} ^{*}$ and $\left.x\right|_{\mathfrak{p}} ^{* *}$ satisfy the definition of $f\left(\mathfrak{p} ; s(\mathfrak{p}), w_{i}\right)$. Hence $\left.x\right|_{\mathfrak{p}} ^{*}=\left.x\right|_{\mathfrak{p}} ^{* *}$.

\section{Conclusion}

In this paper we establish the conditions of rational ethical consumption behaviour and show that a price dependent ethical utility function exists. We propose 
a model of conspicuous ethics in which consumers are not utility maximisers in the usual sense but compete for esteem relative to other members in society. We formulate a model of esteem maximization, where esteem is derived from displaying moral superiority towards society through the consumption of ethical conspicuous goods. Ethical consumers belong to a class of informed consumers who retrieve information via social labels and ethical price systems which do not only perform the role of efficient resource allocation but also inform consumers about the way goods are produced and distributed.

\section{Conflicts of Interest}

The author declares no conflicts of interest regarding the publication of this paper.

\section{References}

[1] Veblen, T. (1899) The Theory of the Leisure. Macmillan, London.

[2] Samuelson, P.A. (1947) Foundations of Economic Analysis. Harvard University Press, Cambridge, MA.

[3] Scitovsky, T. (1945) Some Consequences of the Habit of Judging Quality by Price. Review of Economic Studies, 12, 100-105. https://doi.org/10.2307/2296093

[4] Leibstein, H. (1950) Bandwagon, Snob, and Veblen Effects in the Theory of Consumers' Demand. The Quarterly Journal of Consumer Affairs, 64, 183-207. https://doi.org/10.2307/1882692

[5] Alcaly, R. and Klevprick, A. (1970) Judging Quality by Its Price, Snob Appeal, and the New Consumer Theory. Zeitschrift für Nationalökonomie, 30, 53-64. https://doi.org/10.1007/BF01289992

[6] Kalman, P. (1968) Theory of Consumer Behavior When Prices Enter the Utility Function. Econometrica, 36, 497-510. https://doi.org/10.2307/1909519

[7] Pollak, R. (1977) Price Dependent Preferences. American Economic Review, 67, 64-75.

[8] Lancaster, K. (1971) Consumer Demand: A New Approach to Consumer Theory. Columbia University Press, New York.

[9] Dragusanu, R. and Nunn, N. (2014) The Economics of Fair Trade. Journal of Economic Perspectives, 28, 217-236. https://doi.org/10.1257/jep.28.3.217

[10] Arrow, K. and Hahn, F. (1991) General Competitive Analysis. North Holland, Amsterdam.

[11] Debreu, G. (1959) Theory of Value. Cowles Foundation, New Haven, CT.

[12] Stiefenhofer, P. and Zhang, W. (2020) Conspicuous Ethics: A Veblen Effect Condition for Ethical Consumption Goods. Applied Economics Letters, 28, 217-236. https://doi.org/10.1080/13504851.2020.1855306

[13] Stiefenhofer, P. (2019) Conspicuous Ethical Consumption. Theoretical Economics Letters, 9, 1-8. https://doi.org/10.4236/tel.2019.91001

[14] Sonnenschein, H. (1971) Demand Theory without Transitive Preferences, with Applications to the Theory of Competitive Equilibrium. In: Preferences, Utility, and Demand: A Minnesota Symposium, Harcourt Brace Jovanovich, New York, 215-223.

[15] Blashke, W. (1849) Kreis und Kuge. Chelsea Publishing Company, New York. 